\title{
Acute Herpes Simplex Esophagitis in an Immunocompetent Adult with Eosinophilic Esophagitis
}

\author{
Thomas Adam Wichelmann ${ }^{a}$ Ryan T. Hoff ${ }^{b}$ Dean N. Silas ${ }^{b}$ \\ aDivision of Internal Medicine, Department of Medicine, Advocate Lutheran General \\ Hospital, Park Ridge, IL, USA; bivision of Gastroenterology, Department of Medicine, \\ Advocate Lutheran General Hospital, Park Ridge, IL, USA
}

\section{Keywords}

Eosinophilic esophagitis · Herpes simplex esophagitis · Herpes simplex virus ·

Immunocompetent

\section{Abstract}

Herpes simplex esophagitis (HSE) represents a rare entity in immunocompetent patients and has infrequently been described in association with eosinophilic esophagitis (EoE). Causality in this rare association remains a debated topic. We present a case of HSE occurring in an immunocompetent adult with EoE and review the literature in support of EoE induced mucosal disruption predisposing to increased risk of HSE.

\section{Introduction}

Herpes simplex virus (HSV) is a double-stranded DNA virus that causes mucosal inflammation and ulceration most often associated with gingivostomatitis and pharyngitis [1]. While HSV is known to cause esophagitis in immunocompromised patients, herpes simplex esophagitis (HSE) has also been reported in immunocompetent individuals [1, 2]. Eosinophilic esophagitis (EoE), an esophageal disorder characterized by eosinophil-mediated inflammation and subsequent esophageal dysfunction, has infrequently been described in association with HSE in immunocompetent patients [3]. The association between the 2 conditions is unclear. In vitro and murine skin studies by Thier et al. [4] concluded that functional tight junctions are 
the major physical barrier preventing HSV-1 invasion into tissue and hypothesized that HSV-1 infection requires disruption to intercellular junctions to invade mucosa. Severe, recurrent mucocutaneous HSV infections associated with abnormal desmosomes have been described [5].

In patients with EoE, several histological changes have been observed, including mucosal barrier dysfunction with dilation of intracellular spaces, reduction in tight junction proteins, and abnormal desmosomes with low levels of desmoglein-1 [6]. Tight junction protein levels may increase with histologic remission of EoE [6]. Furthermore, there is evidence that treatment of EoE results in increased expression of the barrier integrity proteins desmoglein-1 and filaggrin, and improved esophageal mucosal integrity [7]. These findings suggest that EoE mucosal disruption may interrupt the integrity of the innate immune system, leading to an increased risk of HSE. We report a case of HSE following EoE and review current literature on potential causal relationships between the conditions.

\section{Case Description}

A 38-year-old man presented to the gastroenterology clinic with a 4-year history of recurrent dysphagia. No past medical or surgical history. No history of recurrent infections. No heartburn. The patient took no medications. Esophagogastroduodenoscopy (EGD) showed esophageal rings and a benign-appearing esophageal stricture, which was dilated to $15 \mathrm{~mm}$ using a through-the-scope balloon dilator. Biopsies of proximal, mid, and distal esophagus showed 40 eosinophils per high-power field, consistent with EoE. He was treated with omeprazole $20 \mathrm{mg}$ BID; symptoms resolved after 6 days of treatment. Additional recommendations included a gluten- and dairy-free diet ( 2 food elimination diet), follow-up in clinic, and repeat EGD in 3 months. Unfortunately, the patient discontinued omeprazole after 3 weeks and did not adhere to dietary or follow-up recommendations.

Four months later, the patient presented to the emergency department with progressive dysphagia for 1 month, chest discomfort and a 3 day history of cold-like symptoms with fatigue and body aches. He described a gradual return of dysphagia for several months, worsening for the 2 weeks prior, until he was unable to swallow solids or liquids. In the emergency department, vital signs showed temperature $37.8^{\circ} \mathrm{C}$, pulse $100 \mathrm{bpm}$, and blood pressure $150 / 105 \mathrm{~mm}$ Hg. Physical exam showed mild acute distress with active regurgitation of food. Laboratory results showed creatinine $1.19 \mathrm{mg} / \mathrm{dL}$, globulin $5.3 \mathrm{~g} / \mathrm{dL}$, blood glucose $117 \mathrm{mg} / \mathrm{dL}$, absent peripheral eosinophils, and mildly elevated peripheral monocytes $(1.3 \mathrm{k} / \mu \mathrm{L})$. Blood tests were otherwise unremarkable.

EGD revealed esophageal rings and exudates in mid and proximal esophagus, along with distal esophagus with ulceration, congestion, friability, and stenosis (Fig. 1). Mid-esophageal

Fig. 1. Endoscopic view of distal esophagus showing esophagitis with ulcerations. Biopsies of this area showed herpes esophagitis without eosinophils.

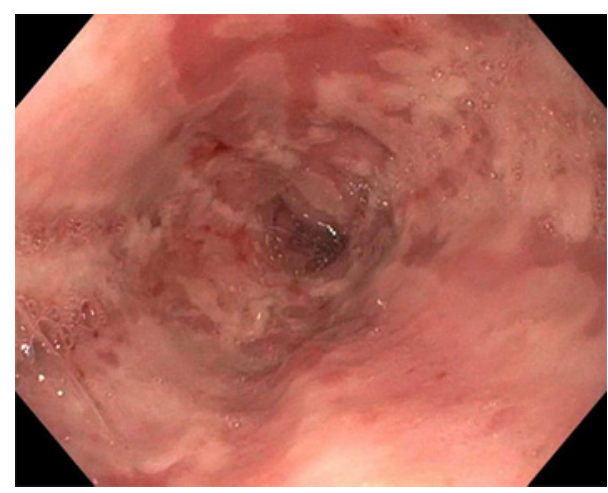


Fig. 2. Histology of distal esophageal biopsy showing marked acute inflammation and numerous multinucleated cells with nuclear viral inclusions, seen at higher power $(\times 40)$. No eosinophils present in this biopsy.

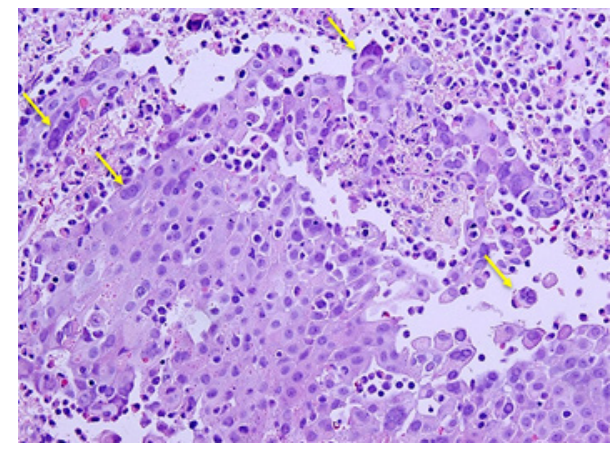

Fig. 3. Immunostain for HSV showing numerous positive viral inclusions.

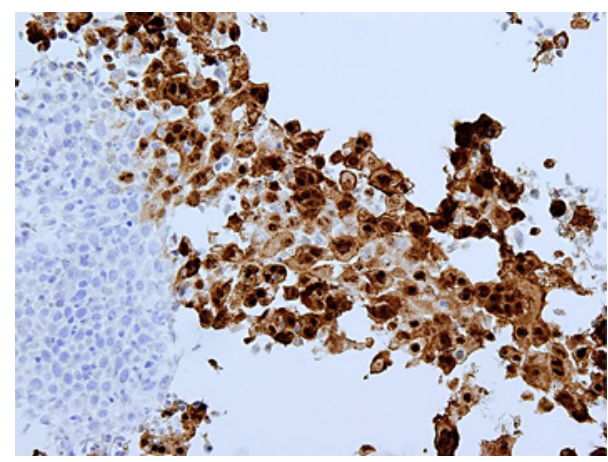

biopsies showed up to 3 eosinophils per high-power field. Distal esophageal biopsies showed ulceration, acute inflammation, and numerous multinucleated cells with nuclear viral inclusions (Fig. 2). No eosinophils were present. Immunostain of distal biopsies was strongly positive for HSV (Fig. 3). The patient was treated with omeprazole $20 \mathrm{mg}$ daily and acyclovir $400 \mathrm{mg}$ 5 times daily for 2 weeks, with resolution of dysphagia. HIV antigen and antibody screen were negative.

\section{Discussion}

We report a rare case of HSE occurring in an immunocompetent adult with incomplete treatment of EoE. After our patient developed a gradual return of dysphagia, eosinophilic inflammation was the expected etiology. However, his dysphagia abruptly worsened with the onset of cold-like symptoms, likely representing the viral prodrome of HSV infection. Esophageal biopsies with immunostain confirmed the diagnosis of HSE. We hypothesize that EoE predisposed our patient to HSE as a result of esophageal barrier dysfunction leading to increased susceptibility to HSV infection [3].

In 2016, Zimmermann et al. [3] described 5 cases of immunocompetent patients with HSV and established or suspected concomitant EoE. Two immunocompetent patients had confirmed EoE prior to presenting with HSE, while a third developed HSE on oral viscous budesonide [3]. HSE may also occur with topical corticosteroid therapy for EoE, possibly due to a local immunosuppressive effect [8]. To our knowledge, our case is the 5th case of HSE in an immunocompetent adult with confirmed EoE reported in English (Zimmerman et al. [3] $\times 2$, Monsanto et al. [9] $\times 1$, Machicado et al. [10] $\times 1$ ), and the third such case where EoE preceded HSV (Zimmerman et al. [3] × 2). 
There are several clinical recommendations that can be considered when approaching patients with EoE and/or HSE. First, the diagnosis of HSE in any immunocompetent patient should always prompt an evaluation to rule-out underlying predisposing factors, including HIV [11]. Second, while antiviral treatment for HSE in immunocompetent patients is not of proven benefit, it may be considered, as evidence suggests it may shorten duration of illness [12]. Third, HSE should be considered in the differential diagnosis for patients with EoE and recurrent dysphagia or odynophagia, particularly with a viral prodrome. This case highlights the importance of completion of EoE therapy and close follow-up, as persistent esophageal barrier dysfunction could predispose to increased vulnerability to HSE. As Zimmerman et al. [3] previously highlighted, full and appropriate EoE treatment may confer protection against the risk of developing concomitant HSE. Recommended treatments include topical steroids, proton pump inhibitor therapy, and dietary modification [13]. Recent evidence suggests that persistent symptoms and endoscopic abnormalities in patients with histologically inactive EoE could be secondary to enduring mast cells [14]. Mast cells are known to disrupt mucosal integrity and could predispose to HSE infection despite evidence of histologically inactive EoE, as in the case we previously described [15].

In conclusion, we present a case of EoE preceding HSE in an immunocompetent adult. This case clinically supports the hypothesis that EoE leads to relative esophageal mucosal disruption, resulting in protective barrier dysfunction and subsequent increased vulnerability to HSE.

\section{Acknowledgments}

The authors thank to Nahren Asado for providing histology images.

\section{Statement of Ethics}

Written and verbal informed patient consent obtained for the publication of the details of their medical case and all accompanying images. Ethics approval/review was not required for a single case report in accordance with Advocate Aurora Health institutional IRB guidelines as this is not viewed as Human Subject Research.

\section{Conflict of Interest Statement}

No conflicts of interest to disclose.

\section{Funding Sources}

No funding was used for this submission.

\section{Author Contributions}

T. Adam Wichelmann wrote the initial manuscript draft. All authors provided substantial contributions, draft revisions, and participated in reviewing the article. All authors approved the final manuscript.

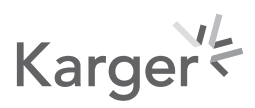




\section{Data Availability Statement}

All data generated or analyzed during this study are included in this article. There is no supplementary material.

\section{References}

1 Eymard D, Martin L, Doummar G, Piché J. Herpes simplex esophagitis in immunocompetent hosts. Can J Infect Dis. 1997;8(6):351-3.

2 Ramanathan J, Rammouni M, Baran J Jr, Khatib R. Herpes simplex virus esophagitis in the immunocompetent host: an overview. Am J Gastroenterol. 2000 Sep;95(9):2171-6.

3 Zimmermann D, Criblez DH, Dellon ES, Bussmann C, Pfeifer D, Froh M, et al. Acute herpes simplex viral esophagitis occurring in 5 immunocompetent individuals with eosinophilic esophagitis. ACG Case Rep J. 2016;3(3): 165-8.

4 Thier K, Petermann P, Rahn E, Rothamel D, Bloch W, Knebel-Mörsdorf D. Mechanical barriers restrict invasion of herpes simplex virus 1 into human oral mucosa. J Virol. 2017 Nov;91(22):1295-317.

5 Vakkilainen S, Puhakka L, Klemetti P, Heiskanen K, Seppanen M, Muona M, et al. Novel DSP spectrin 6 region variant causes neonatal erythroderma, failure to thrive, severe herpes simplex infections and brain lesions. Review Acta Derm Venereol. 2019 Jul 1;99(9):789-96.

6 Blevins CH, Iyer PG, Vela MF, Katzka DA. The esophageal epithelial barrier in health and disease. Clin Gastroenterol Hepatol. 2018;16:608-17.

7 van Rhijn BD, Verheij J, van den Bergh Weerman MA, Verseijden C, van den Wijngaard RM, de Jonge WJ, et al. Histological response to fluticasone propionate in patients with eosinophilic esophagitis is associated with improved functional esophageal mucosal integrity. Am J Gastroenterol. 2015 Sep;110(9):1289-97.

8 Lindberg GM, Eldik RV, Saboorian MH. A case of herpes esophagitis after fluticasone propionate for eosinophilic esophagitis. Nat Clin Pract Gastroenterol Hepatol. 2008 Sep;5(9):527-30.

9 Monsanto P, Almeida N, Cipriano MA, Gouveia H, Sofia C. Concomitant herpetic and eosinophilic esophagitis: a causality dilemma. Acta Gastroenterol Belg. 2012 Sep;75(3):361-3.

10 Machicado JD, Younes M, Wolf DS. An unusual cause of odynophagia in a patient with eosinophilic esophagitis. Gastroenterology. 2014 Jul;147(1):37-8.

11 Canalejo E, García Durán F, Cabello N, García Martínez J. Herpes esophagitis in healthy adults and adolescents: report of 3 cases and review of the literature. Medicine. 2010;89(4):204-10.

12 Kato S, Yamamoto R, Yoshimitsu S, Shimazaki K, Ogawa S, Itoh K, et al. Herpes simplex esophagitis in the immunocompetent host. Dis Esophagus. 2005;18(5):340-4.

13 Hirano I, Chan ES, Rank MA, Sharaf RN, Stollman NH, Stukus DR, et al. AGA Institute and the Joint Task Force on Allergy-Immunology Practice Parameters Clinical Guidelines for the management of eosinophilic esophagitis. Gastroenterology. 2020;158:1776-86.

14 Bolton SM, Kagalwalla AF, Arva NC, Wang MY, Amsden K, Melin-Aldana H, et al. Mast cell infiltration is associated with persistent symptoms and endoscopic abnormalities despite resolution of eosinophilia in pediatric eosinophilic esophagitis. Am J Gastroenterol. 2020 Feb;115(2):224-33.

15 Albert-Bayo M, Paracuellos I, Gonzalez-Castro AM, Rodriguez-Urrutia A, Rodriguez-Lagunas MJ, AlonsoCotoner C, et al. Intestinal mucosal mast cells: key modulators of barrier function and homeostasis. Cells. 2019 Feb;8(2):135.

\section{Karger' ${ }^{\prime \prime}$}

